\title{
Efficient Scheduling for Delay Constrained Multi-Rate CDMA Systems
}

\author{
Min Chen Changyoon Oh Aylin Yener \\ Wireless Communications and Networking Laboratory \\ Electrical Engineering Department \\ The Pennsylvania State University \\ University Park, PA 16802 \\ mchen@psu.edu_cxo155@psu.edu_yener@ee.psu.edu
}

\begin{abstract}
We consider efficient scheduling for a delay constrained multi-rate CDMA system. We first find the optimum schedule for CDMA systems without transmit diversity (TD), that minimizes the average transmission energy per bit while maintaining the short term average throughput for each user in the system. We observe that the specifics of the scheduling problem enables it polynomially solvable. Next, we consider the case when mobile devices are capable of employing two antennas and use Alamouti scheme to achieve TD. We observe that our proposed scheduling protocol can be extended to the system with TD with a modest sacrifice in performance and significant saving in computational complexity, as compared to the optimum scheduler. Simulation results are presented to demonstrate the performance of the proposed scheduling protocols, and the considerable energy savings they provide with respect to the TDMA-type scheduling.
\end{abstract}

\section{INTRODUCTION}

Code Division Multiple Access (CDMA) continues to be the technology of choice for multiuser systems with high data rate and reliability requirements. Efficient transmission strategies are of great interest in any wireless scenario including CDMA systems, due to the limited energy resources as well as delay requirements. There is a tradeoff between energy and delay: by increasing the transmission time, the transmit energy decreases, while the delay increases. Considerable research effort has been devoted to balance this tradeoff and optimize the system performance [1]-[3].

Scheduling plays an important role in enabling efficient transmission for wireless systems with multiple users [4], [5]. Scheduling users in a CDMA system has been investigated to support applications with high bandwidth and strict latency requirements [6], [7]. Energy efficient power and rate allocation for Direct Sequence (DS)-CDMA systems is investigated in [6], whereas reference [7] investigates the optimal and suboptimal solutions to the scheduling problem in systems with multiple receive antennas. These protocols achieve the maximum instantaneous system throughput, however, fairness in terms of the throughput of each user is not considered in either of the references. In many scenarios, we may want to provide fairness among users while optimizing the performance [8]. A short term average throughput guarantee for each user can facilitate fairness. This is the motivation behind our investigation of the efficient scheduling problem with an average throughput requirement.

In this paper, we investigate the efficient scheduling and the resulting power allocation problem for the uplink communications of a delay constrained CDMA system. Specifically, we consider a multi-rate CDMA system facilitated by the aid of multiple codes. Multiple codes belonging to each user become virtual users, and create interference for each other if they transmit at the same time. Our aim is to schedule the virtual users into a given number of time slots with equal duration, such that the total transmission energy is minimized, while the Signal-to-Interference-plus-Noise-Ratio (SINR) target at the base station (BS) is satisfied, and a short term average throughput for each user is guaranteed. At the outset, the problem looks similar to the bin packing problem which is NPcomplete [9]. Fortunately, the specifics of the problem enables it polynomially solvable by a shortest path algorithm.

Next, with the motivation of further improving the performance using multiple antennas, we consider the scheduling problem for the multi-rate CDMA system with transmit diversity (TD) when each user has two transmit antennas that employ the Alamouti scheme [10]. Our numerical results demonstrate that considerable energy savings can be obtained by the proposed schemes with respect to the Time Division Multiple Access (TDMA)-type scheduling scheme, which schedules users in a round robin fashion, i.e., one user transmitting in each slot.

\section{SYSTEM MODEL AND PROBLEM FORMULATION}

We consider the uplink of a single cell CDMA system where $K$ users communicate with a BS. Let $g_{i}$ denote the channel fading coefficient of the $i$ th user to the BS, for $i=1, \ldots, K$. We assume all channels are quasi-static with flat fading, so that the fading coefficients remain constant during a frame of $L$ time slots. Let $\sigma^{2}$ denote the variance of the Additive White Gaussian Noise (AWGN) term at the $\mathrm{BS}^{1}$. Each user may change its transmission rate by the number of spreading codes it uses in each time slot, but maintains the required average rate in a frame. Multiple codes are considered as virtual users, and

${ }^{1} \mathrm{We}$ assume that the out-of-cell interference is included in this term. 
interfere with each other if they transmit in the same time slot, even if they belong to the same user. Each spreading code is assumed to be a randomly generated signature sequence. The BS employs matched filters to decode the users' data from the received signals. The SINR of the $k$ th virtual user of terminal $i$ in time slot $l$ is defined as

$$
S I N R_{i_{k} l}=N \frac{p_{i_{k} l}\left|g_{i}\right|^{2}}{\left(\sum_{j=1}^{K} \sum_{j_{k}=1}^{K_{j l}} p_{j_{k} l}\left|g_{j}\right|^{2}-p_{i_{k} l}\left|g_{i}\right|^{2}\right)+I}
$$

where $p_{j_{k} l}$ denotes the transmit power of the $k$ th virtual user of the $j$ th user in the $l$ th time slot, $K_{j l}$ denotes the number of virtual users of user $j$ in time slot $l$, for $j=1, \ldots, K$ and $l=1, \ldots, L, N$ denotes the processing gain, and $I=N \sigma^{2}$. In the multi-code system, the rate of user $i$ in time slot $l, R_{i l}$, is defined by the number of codes, $K_{i l}$, as

$$
R_{i l}=K_{i l} \times R_{\text {base }}
$$

where $R_{\text {base }}$ is the rate of a virtual user in one time slot, defined as $\frac{W}{N}$, with $W$ as the spreading bandwidth. Thus, the average throughput of user $i, R_{i}$, during a frame of $L$ time slots is

$$
R_{i}=\sum_{l=1}^{L} \frac{R_{i l}}{L}=\sum_{l=1}^{L} \frac{K_{i l} \times R_{\text {base }}}{L}
$$

We aim to minimize the total energy expenditure of all users in a time frame while satisfying the received SINR target, $S I N R_{\text {target }}$, for each virtual user in each slot, and short term throughput requirement, $R_{i_{\text {target }}}{ }^{2}$, for user $i, i=1, \ldots, K$. Given that the time slots in the frame are of equal duration, this problem is equivalent to minimizing the total transmit power of $K$ users in $L$ time slots. The optimization problem can be expressed as

$$
\begin{array}{cc}
\min _{\left\{K_{i l}, p_{i_{k} l}\right\}} & \sum_{l=1}^{L} \sum_{i=1}^{K} \sum_{i_{k}=1}^{K_{i l}} p_{i_{k} l} \\
\text { s. t. } & S I N R_{i_{k} l} \geq S I N R_{\text {target }}, \\
& \forall i_{k}, l \text { such that } p_{i_{k} l}>0 \\
& R_{i}=R_{i_{\text {target }}}, \quad \forall i \\
& p_{i_{k} l} \geq 0, \quad \forall i_{k}, l
\end{array}
$$

Denoting $s_{l}$ as the set of virtual users scheduled in time slot $l$, we have the cardinality of $s_{l}$ as $\left|s_{l}\right|=\sum_{i=1}^{K} K_{i l}$. We note that the optimum received power for each virtual user is achieved when the SINR constraint in (5) is satisfied with equality [11]. Thus, the optimum equal received power for each virtual user in time slot $l$ is

$$
q_{l}^{*}=\frac{I \gamma}{(1+\gamma)-\left|s_{l}\right| \gamma}
$$

where $\gamma=S I N R_{\text {target }} / N$. Since we need $q_{l}^{*}>0$, the maximum number of virtual users in a time slot is limited by $\lfloor(1+\gamma) / \gamma\rfloor$.

Given the relation between the optimum received power and

${ }^{2}$ In other words, we maintain a given number $T_{i}=L R_{i_{\text {target }}} / R_{\text {base }}$ of virtual users for user $i$ during a frame of $L$ time slots. the optimum transmit power, $p_{i_{k} l}^{*}\left|g_{i}\right|^{2}=q_{l}^{*}$, the optimization problem in (4)-(7) can be rewritten as

$$
\begin{array}{ll}
\min _{\left\{K_{i l}\right\}} & \sum_{l=1}^{L} q_{l}^{*} \sum_{i=1}^{K} \frac{K_{i l}}{\left|g_{i}\right|^{2}} \\
\text { s. t. } & \sum_{l=1}^{L} K_{i l}=\frac{n R_{i_{\text {target }}}}{R_{\text {base }}} \quad \forall i
\end{array}
$$

The problem in (9)-(10) is to find $K_{i l}$, the number of virtual users of user $i$ in time slot $l$, for $i=1, \ldots, K$ and $l=1, \ldots, L$, to minimize the total transmit power in $L$ time slots, while each user has $T_{i}=\frac{L R_{i_{\text {target }}}}{R_{\text {base }}}$ virtual users in $L$ time slots. Thus, we have $T=\sum_{i=1}^{K} T_{i}$ virtual users to be scheduled in $L$ time slots such that the total transmit power is minimized.

\section{OPTIMUM SCHEDULE}

In this section, we provide the solution to the optimization problem in (9)-(10). First, we have two observations which give the structure of the optimum scheduling policy.

Observation 1: The optimum policy always schedules a virtual user with a lower channel gain to a time slot with a lighter load, i.e., a slot with fewer virtual users.

To see the validity of observation 1 , we suppose that two virtual users $j$ and $i$ are scheduled to slot 1 and 2 , respectively. We assume that $\left|g_{i}\right|^{2}>\left|g_{j}\right|^{2}$, and $\left|s_{1}\right|>\left|s_{2}\right|$, i.e., slot 1 has more virtual users than slot 2 . If we exchange virtual users $i$ and $j$ between the two slots, all the virtual users except $i$ and $j$ remain the same transmit power level, since $q_{1}^{*}$ and $q_{2}^{*}$ remains the same. However, the sum of the transmit power of $i$ and $j$ is decreased, i.e., $\frac{q_{1}^{*}}{\left|g_{i}\right|^{2}}+\frac{q_{2}^{*}}{\left|g_{j}\right|^{2}}<\frac{q_{1}^{*}}{\left|g_{j}\right|^{2}}+\frac{q_{2}^{*}}{\left|g_{i}\right|^{2}}$. Hence, the total transmit power is decreased.

Observation 1 provides a valuable clue as to the structure of the optimum scheduling policy. Note that, the collection of virtual user sets resulting from any scheduling policy can be reordered as $\left\{s_{1}, s_{2}, \ldots, s_{L}\right\}$, such that $\left|s_{1}\right| \leq\left|s_{2}\right| \leq \ldots \leq\left|s_{L}\right|$. Also, we note that the virtual user sets should satisfy $\left|s_{l}\right| \neq 0$, for $l \in\{1, \ldots, L\}$, and $\sum_{l=1}^{L}\left|s_{l}\right|=T$ for any valid schedule. This reordering of virtual user sets (time slots) does not change the total transmit power expended by this policy. That is, the performance of the scheduling policy is a function of which virtual users are scheduled into each time slot, but not the actual location of these slots in the $L$-slot frame. Therefore, we only need to consider scheduling policies with the reordered virtual user sets to find the optimum solution. Next, we have the following observation.

Observation 2: For any given group of reordered virtual user sets, the optimum scheduling order of $T$ virtual users is in the order of increasing channel gain, i.e.,

$$
\underbrace{\left|g_{K}\right|^{2}, \ldots,\left|g_{K}\right|^{2}}_{T_{K}}, \underbrace{\left|g_{K-1}\right|^{2}, \ldots,\left|g_{K-1}\right|^{2}}_{T_{K-1}}, \ldots, \underbrace{\left|g_{1}\right|^{2}, \ldots,\left|g_{1}\right|^{2}}_{T_{1}}
$$

where $\left|g_{K}\right|^{2} \leq\left|g_{K-1}\right|^{2} \leq \ldots \leq\left|g_{1}\right|^{2}$

Note that the scheduling order in (11) satisfies Observation 1. Hence, (11) is a candidate for the optimum scheduler. Accordingly, the optimization problem in (9)-(10) is to find the best group of reordered virtual user sets such that the sum of the total transmit power is minimized, given the optimum 
scheduling order in (11). By appropriate transformation, this problem can be formulated as a graph partitioning problem whose solution has polynomial complexity, as described next.

The reordered virtual user sets in (11) constitute a string [12], which is a graph where all vertices are located along a line, $G=(V, E)$ with vertices $V=\left\{v_{1}, v_{2}, \ldots, v_{T}\right\}$ and edges $E=\left\{\left(v_{1}, v_{2}\right), \ldots,\left(v_{T-1}, v_{T}\right)\right\}$. Each virtual user is sequentially mapped into the vertex along the string from the left to the right.

Given the string $G$, we denote $\left\{S_{1}, S_{2}, \ldots, S_{L}\right\}$ as the partition of the set of vertices $V$ into $L$ subsets, with each subset $S_{l}$ consisting of a set of connected vertices. Note that in a feasible partition, $\left|S_{l}\right| \neq 0, \forall l \in\{1, \ldots, L\}$. In this setting, the virtual user sets $\left\{s_{1}, s_{2}, \ldots, s_{L}\right\}$ is equivalent to the partition $\left\{S_{1}, S_{2}, \ldots, S_{L}\right\}$. Hence, we use $s_{l}$ and $S_{l}$ interchangeably. The cost of a virtual user set $s_{l}$ is $q_{l}^{*} \sum_{i \in s_{l}} \frac{1}{\left|g_{i}\right|^{2}}$, and the total cost of all virtual user sets is $\sum_{l=1}^{L} q_{l}^{*} \sum_{i \in s_{l}} \frac{1}{\left|g_{i}\right|^{2}}$. Therefore, the optimization problem in (9)-(10) is equivalent to finding a feasible $L$-partition such that the cost is minimized, i.e.,

$$
\begin{aligned}
\min _{\left\{s_{1}, \ldots, s_{L}\right\}} & \sum_{l=1}^{L} q_{l}^{*} \sum_{i \in s_{l}} \frac{1}{\left|g_{i}\right|^{2}} \\
\text { s. t. } & \left|s_{1}\right| \leq\left|s_{2}\right| \leq \ldots \leq\left|s_{L}\right|
\end{aligned}
$$

We note that, although the problem of optimally partitioning an arbitrary graph with an arbitrary cost function is NP-hard, partitioning a string optimally with a separable cost function can be solved in polynomial time [12]. In this case, the problem of graph partitioning of a string can be reduced to a shortest path problem with complexity $O\left(L T^{2}\right)$ [13]. In the following, the solution by a shortest path algorithm is described.

We construct a network from the string $G$ which represents the virtual users. The nodes that lie between the origindestination pair are given by the set

$$
\{(i, j): 1 \leq i \leq L-1 ; i \leq j \leq T-L-1\}
$$

An edge is placed from node $(i 1, j 1)$ to node $(i 2, j 2)$ if $i 2=i 1+1$ and $j 2>j 1$. Otherwise, there is no edge between $(i 1, j 1)$ and $(i 2, j 2)$. There is a one-to-one mapping between the cost function of a feasible partition in a string, and the cost function of a path in the network constructed from the partitioning problem. For node $(i, j), i$ and $j$ denote the index of the time slot and the index of the virtual user, respectively. The cost of a path between nodes $(l-1, t)$ and $(l, t+x)$ is the transmit power cost of the virtual user set $s_{l}$, i.e., $q_{l}^{*} \sum_{i \in s_{l}} \frac{1}{\left|g_{i}\right|^{2}}$, where $x=\left|s_{l}\right|$. We note that the optimum policy should satisfy $\left|s_{1}\right| \leq\left|s_{2}\right| \leq \ldots \leq\left|s_{n}\right|$, and the maximum number of virtual users in a time slot, $\left\lfloor\frac{1+\gamma}{\gamma}\right\rfloor$. Hence, if a path violates any of these two constraints, the cost of the path is set infinite, i.e., the path is infeasible. In Fig. 1, we present an example network constructed from a 5 -vertex string with 3 partition sets, i.e., $T=5$ and $L=3$. Next, a shortest path from the origin to the destination with minimum cost is obtained by using a shortest path algorithm such as Dijkstra's method which has complexity $O\left(L T^{2}\right)$. The

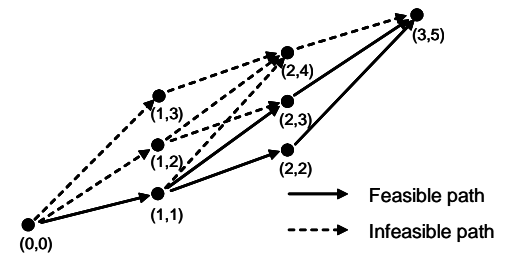

Fig. 1. A network constructed by a 3-partitioning of a 5-vertex string.

resulting optimum partition $\left\{s_{1}, s_{2}, \ldots, s_{L}\right\}$ provides $K_{i l}$, for $i=1, \ldots, K$ and $l=1, \ldots, L$, which is the optimum schedule.

\section{AN EFFICIENT SCHEDULER FOR CDMA SYSTEMS WITH TRANSMIT DIVERSITY (TD)}

Motivated by the increasing demand on utilizing the spatial diversity for wireless systems, in this section, we investigate the scheduling problem for CDMA systems with TD. When there are mobile devices of larger size that have more complicated hardware and more processing capacity, it is feasible to have each user terminal be equipped with two antennas. We will consider this case when Alamouti space-time coding scheme [14] is employed to exploit the spatial diversity.

\section{A. System Model}

We first present the CDMA system model employing the Alamouti scheme [14]. Reference [10] presents a space-time spreading scheme to achieve TD for systems with orthogonal spreading codes. We will assume here a CDMA system with non-orthogonal spreading codes and TD.

Consider a CDMA system with $K$ users and $L$-slot frame. Each user is equipped with two transmit antennas and cooperatively communicates zero-mean independent signals $s_{i 1}$ and $s_{i 2}, i=1, \ldots, K$ with the BS in two time slots. The transmission scheme of user $i$ is shown in Fig. 2. We assume that both antennas of user $i$ have the same transmit power level, i.e., $p_{i 1}=p_{i 2}=p_{i}$, so that the total power is $2 p_{i}$. The channel fading coefficients of the antenna 1 and 2 of user $i$ are denoted by $g_{i 1}$ and $g_{i 2}$, respectively, and $c_{i}$ denotes the randomly generated spreading code of user $i$, for $i=1, \ldots, K$. We next investigate the efficient scheduling protocol for CDMA systems with TD.

\section{B. Scheduling for CDMA Systems with TD}

In this section, we provide the solution to the problem that schedules the transmissions of $K$ users with TD into $L$ time slots. We define the simultaneous transmissions of the signals $s_{i 1}$ and $s_{i 2}$ from user $i$ 's antenna 1 and 2 as a super transmission $T X_{i}$, and the simultaneous transmissions of the conjugate of the signals, i.e., $s_{i 1}^{*}$ and $-s_{i 2}^{*}$ as a super transmission $T X_{i}^{*}, i \in\{1, \ldots, K\}$. Since each user has two super transmissions, each taking one time slot, there are $2 K$ super transmissions to be scheduled into $L$ time slots. We have two observations showing that with some scheduling

\begin{tabular}{c|c|c|c|}
\multicolumn{2}{c|}{} & slot $l_{1}$ & slot $l_{2}$ \\
\hline \multirow{3}{*}{ User i i } & Antenna 1 $\left(g_{i 1}\right)$ & $s_{i 1} c_{i}$ & $-s_{i 2}^{*} c_{i}$ \\
\cline { 2 - 4 } & Antenna 2 $\left(g_{i 2}\right)$ & $s_{i 2} c_{i}$ & $s_{i 1}^{*} c_{i}$ \\
\hline
\end{tabular}

Fig. 2. Transmission scheme of user $i$ with Alamouti scheme. 


\begin{tabular}{|c|c|c|c|c|}
\hline & other slot & slot $l_{1}$ & slot $l_{2}$ & other slot \\
\hline User i & & $T_{i}$ & $T_{i}^{*}$ & \\
\hline User j: case 1 & & $T_{j}$ & $T_{j}^{*}$ & \\
\hline User j: case 2 & & $T_{j}$ & & $T_{j}^{*}$ \\
\hline User j: case 3 & $T_{j}$ & & $T_{j}^{*}$ & \\
\hline User j: case 4 & $T_{j}$ & & & $T_{j}^{*}$ \\
\hline
\end{tabular}

Fig. 3. Possible scheduling schemes of user $j$ with Alamouti scheme.

constraints, the optimum scheduling protocol proposed in Section III is readily applicable to the scheduling problem for the CDMA system with TD at hand.

Observation 3: The super transmission of user $i, T X_{i}$, should not be scheduled into the same time slot with the super transmission $T X_{j}^{*}$ of user $j$, for $i, j \in\{1, \ldots, K\}$.

It is easily seen that if $T X_{i}$, i.e., the transmissions of $s_{i 1}$ and $s_{i 2}$, are scheduled in the same time slot with $T X_{j}^{*}$, i.e., the transmissions of $s_{j 1}^{*}$ and $-s_{j 2}^{*}$, for $i, j \in\{1, \ldots, K\}$, the decoder structure of the Alamouti scheme cannot decouple either $s_{i 1}$ and $s_{i 2}$, or $s_{j 1}$ and $s_{j 2}$, and therefore cannot successfully decode the signals at the BS. Thus, any schedule violates Observation 3 should be avoided.

For any scheduler consistent with Observation 3, let user $i$ be the target user, then the other user $j(j \neq i)$ has four possible schedule schemes as shown in Fig. 3: $\left[\left(T_{j} T_{i}\right),\left(T_{j}^{*} T_{i}^{*}\right)\right]$, $\left[\left(T_{j} T_{i}\right),\left(T_{j}^{*} \mid T_{i}^{*}\right)\right],\left[\left(T_{j} \mid T_{i}\right),\left(T_{j}^{*} T_{i}^{*}\right)\right]$, and $\left[\left(T_{j} \mid T_{i}\right),\left(T_{j}^{*} \mid T_{i}^{*}\right)\right]$, where $(X Y)$ means that super transmissions $X$ and $Y$ are scheduled in the same time slot, and $(X \mid Y)$ means that $X$ and $Y$ are scheduled in different time slots. It is easily shown that the SINR of user $i$, for $i \in\{1, \ldots, K\}$, can be written as

$$
\begin{aligned}
& \operatorname{SINR}_{s_{i 1}}=S I N R_{s_{i 2}} \\
& =\frac{\left|g_{i}\right|^{2} p_{i}}{\frac{1}{N}\left\{A_{i} \sum_{j \in \mathcal{F}_{i}}\left|g_{j}\right|^{2} p_{j}+B_{i} \sum_{j \in \mathcal{L}_{i}}\left|g_{j}\right|^{2} p_{j}\right\}+\sigma^{2}}
\end{aligned}
$$

where $\left|g_{i}\right|^{2}=\left|g_{i 1}\right|^{2}+\left|g_{i 2}\right|^{2},\left|g_{j}\right|^{2}=\left|g_{j 1}\right|^{2}+\left|g_{j 2}\right|^{2}, A_{i}=$ $\left|g_{i 1}\right|^{2} /\left|g_{i}\right|^{2}, B_{i}=\left|g_{i 2}\right|^{2} /\left|g_{i}\right|^{2}$, and $A_{i}+B_{i}=1 . \mathcal{F}_{i}$ denotes the set of users whose $T X_{j}$ are scheduled in the same time slot as $T X_{i}$ of user $i$, and $\mathcal{L}_{i}$ denotes the set of users whose $T X_{j}^{*}$ are scheduled in the same time slot as $T X_{i}^{*}$ of user $i$.

We note that for the SINR given in (15) for CDMA with $\mathrm{TD}$, we lose the form given in Section III, and the scheduler with polynomial complexity is no longer guaranteed. However, when $\mathcal{F}_{i}=\mathcal{L}_{i}$, the SINR in (15) is reduced to

$$
S I N R_{s_{i 1}}=S I N R_{s_{i 2}}=\frac{\left|g_{i}\right|^{2} p_{i}}{\frac{1}{N} \sum_{j \in \mathcal{F}_{i}}\left|g_{j}\right|^{2} p_{j}+\sigma^{2}}
$$

Note that (16) is in the same form as (1). Thus, we have the following observation.

Observation 4: The problem of scheduling $2 K$ super transmissions into $L$ time slots for the CDMA system with TD is equivalent to scheduling a $K$-user CDMA system without TD in $L / 2$ time slots $^{3}$, given that each CDMA user with TD is considered as a single-antenna user with the equivalent channel

\footnotetext{
${ }^{3}$ It is assumed that $L$ is an even integer, i.e., the delay requirements can accommodate up to one wasted slot if necessary.
}

gain $\left|g_{i}\right|^{2}=\left|g_{i 1}\right|^{2}+\left|g_{i 2}\right|^{2}$, and the two time slots it takes are bounded together into one.

With Observation 4, we note that the scheduling protocol proposed in Section III becomes a near-optimum solution to the CDMA systems with TD, by excluding the scheduling schemes of case 2 and 3 in Fig. 3, and it can significantly reduce the computational cost of finding the optimum schedule with acceptable performance penalty, shown in Section $\mathrm{V}$ by numerical results. Note that same results can be directly applied to a multi-rate CDMA system with TD as well.

\section{NUMERICAL RESULTS}

In this section, we present numerical results related to the performance of the efficient scheduling protocols. We consider the uplink of a CDMA system consisting of $K$ users randomly distributed in a circle area with radium $r$, and a BS located in the center of the circle, with spreading bandwidth $W=$ $1.228 \mathrm{MHz}$, and the processing gain $N=128$, equivalently, $R_{\text {base }}=9.6 K b p s$. The transmission frame duration is $L$ time slots. The fading coefficient of user $i, g_{i}$, is modeled as independent complex Gaussian with variance $\sigma_{g_{i}}^{2}=C / d_{i}^{\alpha}$, where $d_{i}$ denotes the distance between user $i$ and the BS. For the CDMA system with TD, we assume the distances from the two antennas of user $i$ to the BS are the same, also denoted by $d_{i}$, and therefore the fading coefficients of the two antennas are independent complex Gaussian with the same variance, i.e., $\sigma_{g_{i 1}}^{2}=\sigma_{g_{i 2}}^{2}=C / d_{i}^{\alpha}$. The path-loss exponent is denoted by $\alpha$, and $C$ is a constant. The values $\alpha=3, C=7 \times 10^{-4}$, and $S I N R_{\text {target }}=7 d B$ are used throughout our simulations. The AWGN variance is assumed to be $10^{-13}$.

Simulation results are presented to demonstrate the performance of the proposed scheduling protocols, compared with the TDMA-type schedule, in which user $i$ transmits with rate $n R_{i_{\text {target }}}$, and all users transmit in a round robin fashion, i.e., only one user transmits in one time slot. Specifically, we plot the average transmit energy per bit, versus the average throughput requirement at each user.

We first compare the performance between the optimum schedule and the TDMA-type schedule for a CDMA system without TD. There are $K=20$ users, and radium of the distribution area is $r=100 \mathrm{~m}$. The transmission frame consists of 5 slots. Fig. 4 shows the average energy consumption per bit for a common average throughput requirement at each user as $R_{i_{\text {target }}}=\{1.92 \mathrm{Kbps}, 3.84 \mathrm{Kbps}, 5.76 \mathrm{Kbps}, 9.6 \mathrm{Kbps}\}$, for $i=1, \ldots, 20$. We observe that a substantial amount of energy is saved by employing the optimum scheduling protocol, with respect to the TDMA-type schedule. As the average throughput requirement increases, i.e., the users loads get heavier, the gap between the performance of the optimum scheduling and that of the TDMA-type schedule increases. This result clearly indicates the benefit of the optimum schedule for a loaded CDMA system.

We also investigate the performance of the schedule for the CDMA system with TD. We compare the performance of the optimum and the near-optimum schedule for systems consisting of $K=\{4,5,6\}$ users, each taking 2 slots to 


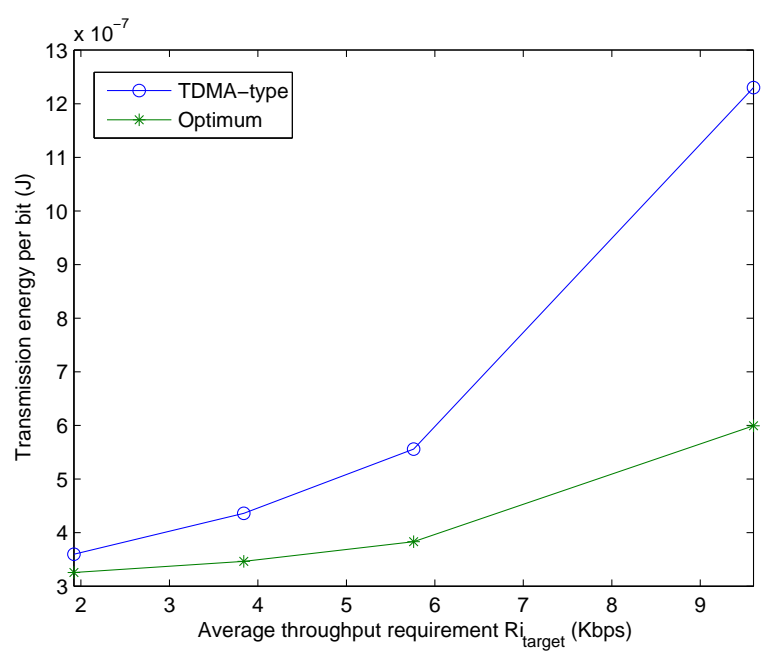

Fig. 4. Average energy consumption of the CDMA system without TD.

transmit and having the average throughput requirement $R_{i_{\text {target }}}=9.6$ Kbps. The transmission frame consists of 6 slots. Table I shows that the near-optimum schedule incurs less than $10 \%$ performance penalty while providing a significantly reduction in computational complexity compared to the optimum schedule, which is achieved by exhaustive search. Next, we consider a larger system with $K=5$ users and the distribution area with $r=1000 \mathrm{~m}$. The frame duration is 10 slots. The common average throughput requirements for the users are $R_{i_{\text {target }}}=$ $\{3.84 K$ bps, 7.68 Kbps, 11.52 Kbps, 15.36 Kbps, 19.2 Kbps $\}$, for $i=1, \ldots, 5$. For the case without TD, we assume that each user is equipped with single antenna and no TD is exploited. Comparing the performance with and without TD as shown in Fig. 5, we observe that a large amount of energy is saved by TD. At the same time, by employing the scheduling protocol, more energy is saved by the near-optimum schedule with respect to the TDMA-type schedule.

\section{CONCLUSIONS}

In this work, we have considered efficient scheduling strategies for delay constrained multi-rate CDMA systems. Short term average throughput requirements are imposed to maintain an average throughput in addition to the QoS requirements (SINR target) for each user. It is assumed that multiple data rates are provided by means of multiple spreading codes, each of which is treated as a virtual user and interferes with each other when transmitting simultaneously. We have provided the optimum scheduler which can be found in polynomial time by solving a shortest path problem. We have further

TABLE I

COMPARISON OF THE OPTIMUM AND NEAR-OPTIMUM SCHEDULER ON

AVERAGE TRANSMIT ENERGY PER BIT.

\begin{tabular}{|c|l|l|l|}
\hline User number & $\begin{array}{l}\text { Optimum } \\
\left(10^{-5} \mathrm{~J} / \text { bit }\right)\end{array}$ & $\begin{array}{l}\text { Near-optimum } \\
\left(10^{-5} \mathrm{~J} / \text { bit }\right)\end{array}$ & Penalty \\
\hline 4 & 4.3091 & 4.4826 & $4.03 \%$ \\
\hline 5 & 4.3217 & 4.6165 & $6.82 \%$ \\
\hline 6 & 4.3267 & 4.6839 & $8.26 \%$ \\
\hline
\end{tabular}

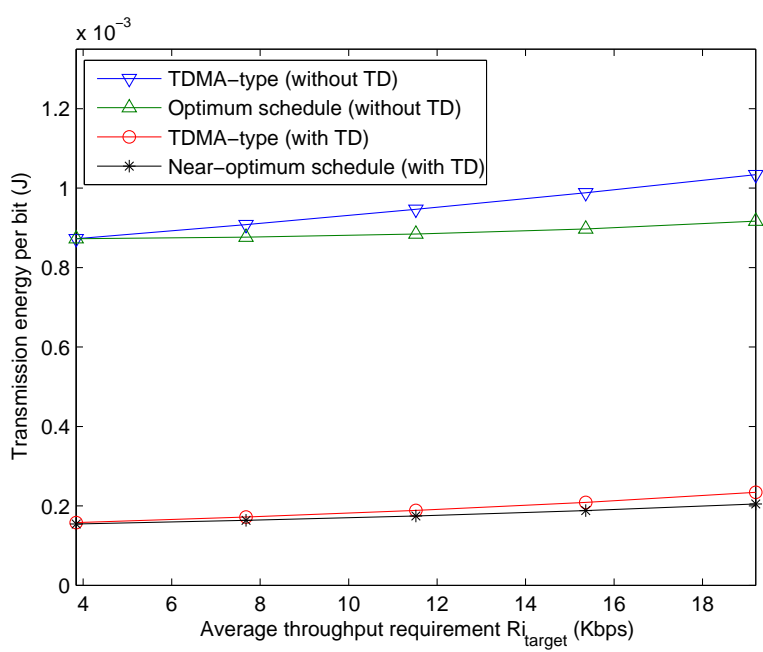

Fig. 5. Average energy consumption of the CDMA system with TD.

considered CDMA systems with mobile terminals that have two transmit antennas, and showed that with some conditions, the proposed scheduling algorithm can be applied directly to CDMA systems with TD yielding a near-optimum scheduler. The numerical results demonstrate that significant energy savings is achieved by the proposed scheduling protocols.

\section{REFERENCES}

[1] R. A. Berry and R. G. Gallager. Communication over fading channels with delay constraints. IEEE Transactions on Information Theory, 48(5):1135 - 1149, May 2002.

[2] E. Uysal-Biyikoglu, B. Prabhakar, and A. El Gamal. Energy-efficient packet transmission over a wireless link. IEEE/ACM Transactions on Networking, 10(4):487 - 499, August 2002.

[3] A. Fu, E. Modiano, and J. Tsitsiklis. Optimal energy allocation for delay-constrained data transmission over a time-varying channel. In IEEE INFOCOM'03, March 2003.

[4] S. Cui, R. Madan, A. J. Goldsmith, and S. Lall. Energy-delay tradeoffs for data collection in TDMA-based sensor networks. In IEEE International Conference on communications, ICC'05, May 2005.

[5] Y. Yao and G. B. Giannakis. Energy-efficient scheduling protocols for wireless sensor networks. In IEEE International Conference on Communications, ICC'05, May 2005.

[6] F. Berggren and Seong-Lyun Kim. Energy-efficient control of rate and power in DS-CDMA systems. IEEE Transactions on Wireless Communications, 3(3):725 - 733, May 2004.

[7] Il-Min Kim, R. Yim, and H. Chaskar. Optimum scheduling for smart antenna systems in Rayleigh fading channels. IEEE Transactions on Communications, 53(7):1210 - 1219, July 2005.

[8] A. K. Dinnis and J. S. Thompson. Investigation of fairness issues with multiple antennas in high data rate CDMA systems. In IEEE Vehicular Technology Conference, VTC'04 Spring, pages 723 - 727, May 2004.

[9] M. R. Garey and D. S. Johnson. A guide to the theory of NPcompleteness. W. H. Freeman and company, 1991.

[10] B. Hochwald, T. L. Marzetta, and C. B. Papadias. A transmitter diversity scheme for wideband CDMA systems based on space-time spreading. IEEE Journal on Selected Areas in Communications, 19(1):48 - 60, January 2001.

[11] R. D. Yates. A framework for uplink power control in cellular radio systems. IEEE Journal on Selected Areas in Communications, 13(7):1341 - 1347, September 1995.

[12] R. Diestel. Graph Theory. Springer, 1997.

[13] C. De Simone, M. Lucertini, S. Pallottino, and B. Simeone. Fair dissections of spiders, worms, and caterpillars. Networks, 20:323 - 344, 1990.

[14] S. M. Alamouti. A simple transmit diversity technique for wireless communications. IEEE Journal on Selected Areas in Communications, 16(8):1451 - 1458, October 1998. 